\title{
Topological Representation of Contact Lattices
}

\author{
Ivo Düntsch ${ }^{\star 1}$, Wendy MacCaull ${ }^{\star 2}$, Dimiter Vakarelov ${ }^{\star \star 3}$, and Michael \\ Winter ${ }^{\star 1}$ \\ 1 Department of Computer Science \\ Brock University \\ St. Catharines, ON, Canada \\ duentsch,mwinter@brocku.ca \\ 2 Department of Mathematics, Statistics and Computer Science \\ St. Francis Xavier University \\ Antigonish, NS, Canada \\ wmaccaul@stfx.ca \\ 3 Department of Mathematical Logic \\ Sofia University \\ Sofia, Bulgaria \\ dvak@fmi.uni-sofia.bg
}

\begin{abstract}
The theory of Boolean contact algebras has been used to represent a region based theory of space. Some of the primitives of Boolean algebras are not well motivated in that context. One possible generalization is to drop the notion of complement, thereby weakening the algebraic structure from Boolean algebra to distributive lattice. The main goal of this paper is to investigate the representation theory of that weaker notion, i.e., whether it is still possible to represent each abstract algebra by a substructure of the regular closed sets of a suitable topological space with the standard Whiteheadean contact relation.
\end{abstract}

\section{Introduction}

In the classical approach to space the basic primitive is the notion of a point, and geometric figures are considered to be sets of points. Contrary to this, the regionbased approach to space adopts as its primitives more realistic spatial notions. In this theory, regions, as abstraction ofs "solid" spatial bodies, and several basic relations and operations between regions are considered. Some of the relations have their origin in mereology, e.g. "part-of" $(x \leq y)$, "overlap" $(x O y)$, its dual "underlap" $(x U y)$ and others definable by them. Region based theory of space extends classical mereology by considering some new relations between regions, topological in nature, such as "contact" $(x C y)$, "non-tangential partof" $(x \ll y)$ and many others definable mainly by means of the contact and the

\footnotetext{
* The author gratefully acknowledges support from the Natural Sciences and Engineering Research Council of Canada.

** This author was supported by the project NIP-1510 by the Bulgarian Ministry of Science and Education
} 
part-of relations. This motivates some authors to call the new direction "mereotopology". The most simple algebraic counterpart of mereotopology are contact algebras, appearing in different papers under various names (see for instance $[4-9,13,14]$ ), which are Boolean algebras extended with the contact relation $C$ satisfying some axioms. The elements of the Boolean algebra represent regions and the Boolean operations - join $x+y$, meet $x \cdot y$ and the Boolean complement $x^{*}$ - allow the construction of new regions from given ones (such as the "universal" region 1 , having all other regions as its parts, and the zero region 0 , which is part of all regions). Part-of, underlap and overlap are definable by the Boolean part of the algebra, i.e., $x \leq y$ is the lattice ordering, $x O y \leftrightarrow x \cdot y \neq 0$ (motivating the name "overlap") and $x U y \leftrightarrow x+y \neq 1$ (motivating the name "underlap"). So, the Boolean part of a contact algebra incorporates the mereological aspect of the theory, while the contact relation $C$ corresponds to the "topological" part of the formalism. The word "topology" in this context is well motivated, because standard models of contact algebras are the Boolean algebras of regular closed (or regular open) sets of a given topological space. We recall that $a$ is regular closed if $a=C l(\operatorname{Int}(a))$, and, dually, $c$ is regular open if $c=\operatorname{Int}(C l(c))$, where $C l$ and Int are the topological operations of closure and interior in the topological space. Note that in either case the Boolean operations are not the standard set-theoretic operations, i.e., we have $a \cdot b=C l(\operatorname{Int}(a \cap b)), a+b=a \cup b$, and $a^{*}=C l(-a)$ for regular closed sets $a$ and $b$, and $c \cdot d=c \cap d, c+d=\operatorname{Int}(C l(c \cup d))$, and $c^{*}=\operatorname{Int}(-c)$ for regular open sets $c$ and $d$. The contact relation between regular closed regions is given by $a C b \leftrightarrow a \cap b \neq \varnothing$ and for open regions by $c C d \leftrightarrow C l(c) \cap C l(d) \neq \varnothing$. Topological contact algebras correspond to the pointbased aspect of the theory.

One of the main streams in the theory of contact algebras is their topological representation theory, i.e., the construction of an embedding of the contact algebra into a suitable topological space. In addition, one usually requires that the embedding generates/determines the topology. For Boolean contact algebras it is natural to require that the image of the embedding is a basis for the topology (similar to the Stone representation theory for Boolean algebras). In the case of distributive lattices this approach works if and only if underlap satisfies an additional property (Corollary 2 and Theorem 5). By relaxing that strict interpretation of "generate the topology" we get a general representation theorem for distributive contact algebras (Theorem 7). Once such a theorem is established, it shows that, although the notion of point is not a primitive notion of the theory, it can be defined within the algebra via its isomorphic copy in the topological contact algebra provided by the representation theorem. This shows that the "pointless" abstraction is adequate and corresponds to the classical point-based approach. Recent results in this direction can be found in $[4,5,8$, 14]. For instance, in [4] several versions of abstract points for contact algebras are discussed such as clans, E-filters etc. All versions generalize the notion of an ultrafilter, which is the abstract point suitable for representing Boolean algebras as ordinary sets. Let us note that ordinary sets can also be considered as regions in a topological space endowed with the discrete topology, but such regions are 
"bad" regions in the sense that they do not have nice topological properties such as a boundary, an non-tangential part etc.

One of the main goals of this paper is to generalize the notion of contact algebras by weakening the algebraic structure from Boolean algebras to distributive lattices, but to keep the intended semantics of regions to be regular sets in topological spaces. In other words, we simply remove the operation of Boolean complement * From a philosophical point of view, the complement of a region is not well motivated. If the region $a$ represents a solid body what is then represented by $a^{*}$ ? (One can formulate similar criticisms for (certain aspects) of some of the other Boolean primitives, which are not discussed here.) Notice that the definitions of the mereological relations part-of, overlap and underlap do not depend on the existence of complements. Moreover, in all known definitions and variations of Boolean contact algebras, the axioms for contact do not rely on complements. So, studying a theory based on weaker assumptions will reveal more deeply the nature of the mereological and mereo-topological relations. The mereo-topological relations usually considered such as "non-tangential part" $x \ll y \leftrightarrow a \bar{C} b^{*}$ and dual contact $a \check{C} b \leftrightarrow a^{*} C b^{*}$ are definable from contact using complements. In the case of distributive lattices these relations must be primitives. Using this approach a deeper insight into the separate roles of the different mereological relations and their interactions may be achieved. For instance, in the Boolean case a certain mereological relation may possess some properties, which must be postulated separately for distributive lattices. An example is the property, " $U$ is extensional", which implies that part-of is definable by $U$ in the sense that $a \leq b$ if and only if $(\forall c)(b U c \rightarrow a U c)$. It turns out (Corollary 2) that this property is exactly the necessary and sufficient property for representing the contact structure in the strict sense. On the other hand, for Boolean contact algebras such a representation is always possible, because in that case underlap is extensional.

The paper is organized as follows. Section 2 introduces the algebraic notions such as distributive lattices, overlap and underlap, distributive contact algebras, filters and clans. Section 3 provides the topological background and a pure topological theorem relating extensionality of underlap to the generation of the topology by means of regular closed sets. It also shows the necessity of the distributivity of the lattice. In Section 4 we show how to represent U-extensional distributive contact lattices in a lattice of regular closed sets of some topological space. As a side result we obtain Cornish's theorem [3] for U-extensional distributive lattices. Here we prove also that every distributive contact lattice can be embedded in a lattice of regular closed sets, so that the image of the lattice generates the topology in a weaker sense. This is done in two steps. First we show that every distributive contact lattice can be embedded into a U-extensional contact lattice; then, we simply apply the representation theorem for $U$-extensional contact lattices. The last section contains some conclusions and future work.

A standard reference for distributive lattices is [2] and for topology [10]. 


\section{Notation and first observations}

For any set $X$ and $Y \subseteq X$ we denote by $-Y$ the complement of $Y$ in $X$, if no confusion can arise. If $R$ is a binary relation on $D$, and $x \in D$, we let $R(x)=\{y: x R y\}$, which is called the range of $x$ with respect to $R$.

\section{Distributive lattices}

Throughout this paper, $\langle D, 0,1,+, \cdot\rangle$ is a bounded distributive lattice; we usually denote algebras by their base set. The dual lattice $D^{o p}$ of $D$ is the lattice $(D, 1,0, \cdot,+)$ based on the reversed order of $D$. A sublattice $D^{\prime}$ of $D$ is called dense (in $D$ ) iff for each element $0 \neq a \in D$ there is an element $0 \neq b \in D^{\prime}$ with $b \leq a$. A dually dense sublattice of $D$ is a dense sublattice of $D^{o p}$. Finally, we call an embedding $h: D^{\prime} \rightarrow D$ dense iff the image $h\left(D^{\prime}\right)=\left\{h(a): a \in D^{\prime}\right\}$ of $D^{\prime}$ is dense in $D$.

We define two relations on $D$, which are of importance in things to come:

$$
\begin{array}{ll}
x O y \Longleftrightarrow x \cdot y \neq 0, & \text { "overlap", } \\
x U y \Longleftrightarrow x+y \neq 1, & \text { "underlap". }
\end{array}
$$

The proof of the following is straightforward:

Lemma 1. If $a \leq b$, then $O(a) \subseteq O(b)$ and $U(b) \subseteq U(a)$.

$O$ is called extensional if

$$
(\forall x, y)[O(x)=O(y) \Rightarrow x=y] .
$$

Analogously, we say that $U$ is extensional if

$$
(\forall a, b)[U(a)=U(b) \Rightarrow a=b]
$$

In [15] distributive lattices which satisfy (2.3) are called disjunctive lattices. If $D$ is a Boolean algebra, then, clearly, both $O$ and $U$ are extensional. Extensionality of $O$ and $U$ has been considered earlier in the literature, and these results show that such extensionalities can influence the underlying algebraic structure; in particular, the following holds for a bounded distributive pseudocomplemented lattice (i.e., a bounded distributive lattice equipped with an operation * satisfying $a \leq b^{*}$ iff $\left.a \cdot b=0\right)$ :

Theorem 1. 1. Suppose that $D$ is a bounded distributive pseudocomplemented lattice. Then, $D$ is a Boolean algebra if and only if $O$ is extensional.

2. Suppose that $D$ is a bounded distributive dually pseudocomplemented lattice. Then, $D$ is a Boolean algebra if and only if $U$ is extensional.

Proof. 1. was shown in [9], and 2. follows by duality. 
In particular, if $D$ is finite and not a Boolean algebra, or if $D$ is a chain, then neither $O$ nor $U$ is extensional. Furthermore, if 0 is meet irreducible then $O$ is not extensional, and if 1 is join irreducible, then $U$ is not extensional. For example, the lattice of all cofinite subsets of $\omega$ together with $\emptyset$ is $U$-extensional, but not $O$-extensional; dually, the set of all finite subsets of $\omega$ together with $\omega$ is $O$-extensional, but not $U$-extensional.

In [3] further characterizations of disjunctive and dually disjunctive lattices were given; these relate to dense sublattices of Boolean algebras:

Theorem 2. 1. $O$ is extensional if and only if $D$ is isomorphic to a dense sublattice of a complete Boolean algebra.

2. $U$ is extensional if and only if $D$ is isomorphic to a dually dense sublattice of a complete Boolean algebra.

3. $O$ and $U$ are both extensional if and only if the Dedekind completion of $D$ is a complete Boolean algebra.

Below, we will give additional conditions for $O$, respectively $U$, to be extensional. It is worthy of mention that each of these is strictly stronger than extensionality in the case that $D$ is not distributive [9].

Lemma 2. 1. $O$ is extensional if and only if $(\forall a, b)[O(a) \subseteq O(b) \Rightarrow a \leq b]$.

2. $U$ is extensional if and only if $(\forall a, b)[U(b) \subseteq U(a) \Rightarrow a \leq b]$.

Proof. 1. " $\Rightarrow ":$ Suppose that $O(a) \subseteq O(b)$. Then, $O(b)=O(a) \cup O(b)=$

$O(a+b)$. Extensionality of $O$ implies that $b=a+b$, i.e. $a \leq b$.

"Æ": Let $O(a)=O(b)$; then $O(a) \subseteq O(b)$ and $O(b) \subseteq O(a)$, and, by the hypothesis, $a \leq b$ and $b \leq a$, i.e., $a=b$.

2 . is proved dually.

Later, we use extensionality in the equivalent form given by Lemma 2 . If, for instance, $U$ is extensional then we will say that the lattice is U-extensional.

A subset $F$ of a lattice $D$ is called a filter if $x, y \in F$ and $z \in D$ implies $x \cdot y \in F$ and $x+z \in F$. We call a filter $F$ of $D$ prime if $x+y \in F$ implies $x \in F$ or $y \in F$. Prime $(D)$ is the set of prime filters of $D$. For each $x \in D$ we denote by $h_{\text {Prime }}(x)=\{F \in \operatorname{Prime}(D): x \in F\}$, the set of all prime filters containing $x$. Stone's well known representation theorem now states:

Theorem 3. [2, 12]

1. The mapping $h_{\text {Prime }}$ is a lattice embedding of $D$ into the lattice $2^{\text {Prime }(D)}$ of all subsets of Prime $(D)$.

2. The collection $\left\{h_{\text {Prime }}(a): a \in D\right\}$ forms a basis for the closed sets of a compact $T_{0}$ topology $\tau$ on Prime $(D)$ for which each set $($ Prime $(D) \backslash h(a))$ is compact open. Furthermore, $\tau$ is a $T_{1}$ topology if and only if $D$ is relatively complemented, and a $T_{2}$ topology if and only if $D$ is a Boolean algebra.

For later use we observe that $h_{\text {Prime }}(a)$ is not necessarily regular closed. 


\section{Contact relations and distributive contact lattices}

A binary relation $C$ on $D$ is called a contact relation $(\mathrm{CR})$ if it satisfies:

C0. $(\forall a) 0(-C) a$;

C1. $(\forall a)[a \neq 0 \Rightarrow a C a]$;

C2. $(\forall a)(\forall b)[a C b \Rightarrow b C a]$;

C3. $(\forall a)(\forall b)(\forall c)[a C b$ and $b \leq c \Rightarrow a C c]$;

C4. $(\forall a)(\forall b)(\forall c)[a C(b+c) \Rightarrow(a C b$ or $a C c)]$.

The pair $\langle D, C\rangle$ is called a distributive contact lattice (DCL). If $D$ is a Boolean algebra, then $\langle D, C\rangle$ is a Boolean contact algebra (BCA). Let $\mathcal{C}$ denote the set of contact relations on $D$. The next lemma shows that $\mathcal{C}$ is not empty.

Lemma 3. $O$ is the smallest contact relation on $D$.

Proof. Suppose that $C \in \mathcal{C}$. If $x \cdot y \neq 0$, then $(x \cdot y) C(x \cdot y)$ by C1, and $\mathrm{C} 3$ now implies that $x C y$.

An extensive investigation of lattices of contact relations on a Boolean algebra is provided by [7].

In the next lemma we relate a contact relation to products of prime filters:

Lemma 4. If $C \in \mathcal{C}$, then $C=\bigcup\{F \times G: F, G \in \operatorname{Prime}(D), F \times G \subseteq C\}$.

Proof. This was proved in [6] for Boolean contact lattices, and an analysis of the proof shows that it also holds for distributive contact lattices.

A clan is a nonempty subset $\Gamma$ of $D$ which satisfies:

CL1. If $x, y \in \Gamma$ then $x C y$;

CL2. If $x+y \in \Gamma$ then $x \in \Gamma$ or $y \in \Gamma$;

CL3. If $x \in \Gamma$ and $x \leq y$, then $y \in \Gamma$.

Note that each prime filter is a clan. The set of all clans of $D$ will be denoted by $\operatorname{Clan}(D)$.

Corollary 1. $a C b$ iff there exists a clan $\Gamma$ such that $\{a, b\} \subseteq \Gamma$.

Proof. Suppose that $a C b$; by the previous Lemma, there are $F, G \in \operatorname{Prime}(D)$ such that $a \in F, b \in G$, and $F \times G \subseteq C$. Clearly, $F \cup G$ is a clan containing both $a$ and $b$. The converse follows from the definition of clan.

\section{Topological models}

First we want to recall some notions from topology. By a topological space $(X, C(X))$ we mean a set $X$ provided with a family $C(X)$ of subsets, called closed sets, which contains the empty set $\varnothing$ and the whole set $X$, and is closed with respect to finite unions and arbitrary intersections. The system $(C(X), \varnothing, X, \cap, \cup)$ 
is a distributive lattice, called the lattice of closed sets of $X: \varnothing$ is the zero element and $X$ is the unit element of the lattice and the set inclusion is the lattice ordering. Fixing $C(X)$ we say that $X$ is endowed with a topology. A subset $a \subseteq X$ is called open if it is the complement of a closed set. The family $O p(X)$ of open sets of $X$ is also a lattice with respect to the same operations. A family of closed sets $\mathbf{B}(X)$ is called a closed basis of the topology if every closed set can be represented as an intersection of sets from $\mathbf{B}(X)$. Consequently, $X \in \mathbf{B}(X)$ and $B(X)$ is closed under finite unions; hence, $(\mathbf{B}(X), X, \cup)$ is an upper semi-lattice. Finally, a family of closed sets $B$ is called a (closed) sub-basis of the topology if the set of finite unions of elements of $B$ is a closed basis.

In every topological space one can define the following operations on subsets $a \subseteq X:$

1. $C l(a)=\bigcap\{c \in C(X): a \subseteq c\}$ (the closure of $a$ ), i.e., the intersection of all closed sets containing $a$.

2. Int $(a)=\bigcup\{o \in O p(X): a \subseteq o\}$ (the interior of $a$ ), i.e., the union of all open sets contained in $a$.

$C l$ and $\operatorname{Int}$ are interdefinable, i.e. $C l(a)=-\operatorname{Int}(-a)$ and $\operatorname{Int}(a)=-C l(-a)$. If $\mathbf{B}(X)$ is a closed base of $X$, then obviously:

$$
C l(a)=\bigcap\{b \in \mathbf{B}(X): a \subseteq b\} .
$$

The next two facts follows from above:

$$
\begin{gathered}
x \in C l(a) \text { iff }(\forall b \in \mathbf{B}(X))(a \subseteq b \rightarrow x \in b) ; \\
x \in \operatorname{Int}(a) \text { iff }(\exists b \in \mathbf{B}(X))(a \subseteq b \text { and } x \notin b) .
\end{gathered}
$$

A subset $a$ of $X$ is called regular closed if $C l(\operatorname{Int}(a))=a$, and, dually, regular open if $\operatorname{Int}(C l(a))=a$ (in this paper we will mainly work with regular closed sets). We denote by $R C(X)$ the family of regular closed sets of $X$. It is a well known fact that $R C(X)$ is a Boolean algebra with respect to the following operations and constants:

$$
0=\varnothing, 1=X, a+b=a \cup b \text { and } a \cdot b=C l(\operatorname{Int}(a \cap b)) .
$$

$R C(X)$ naturally provides a contact relation $C$ defined by $a C b$ iff $a \cap b \neq \varnothing$. $C$ is called the standard (or Whiteheadean) contact relation on $R C(X)$.

A topological space is called semi-regular if it has a closed base of regular closed sets.

Every topological space $X$ can be made semi-regular by defining a new topology taking as a base, the set $R C(X)$. It is a well known fact that this new topology generates the same set of regular closed sets.

The following topological theorem gives necessary and sufficient conditions for a closed base of a topology to be semi-regular.

Theorem 4. [Characterization theorem for semi-regularity] Let $X$ be a topological space and $\mathbf{B}(X)$ be a closed base for $X$. Suppose that . is a binary operation defined the set $\mathbf{B}(X)$ so that $(\mathbf{B}(X), \varnothing, X, \cup, \cdot)$ is a lattice (not necessary distributive). Then we have: 
1. The following conditions are equivalent:

(a) $\mathbf{B}(X)$ is $U$-extensional.

(b) $\mathbf{B}(X) \subseteq R C(X)$.

(c) For all $a, b \in \mathbf{B}(X), a \cdot b=C l(\operatorname{Int}(a \cap b))$.

(d) $(\mathbf{B}(X), \varnothing, X, \cup, \cdot)$ is a dually dense sublattice of the Boolean algebra $R C(X)$.

2. If any of the (equivalent) conditions $(a),(b),(c)$ or $(d)$ of 1 . is fulfilled then:

(a) $(\mathbf{B}(X), \varnothing, X, \cup, \cdot)$ is a $U$-extensional distributive lattice.

(b) $X$ is a semi-regular space.

Proof. 1. (a) $\rightarrow(\mathbf{b})$. Let $\mathbf{B}(X)$ be $U$-extensional, i.e., for all $a, b \in \mathbf{B}(X)$ the following holds:

$$
(\forall c \in \mathbf{B}(X))(a \cup c=X \rightarrow b \cup c=X) \rightarrow a \subseteq b .
$$

We must show that for every $a \in \mathbf{B}(X), a=C l(\operatorname{Int}(a))$. This follows from the following chain of equivalences:

$$
\begin{aligned}
& x \in C l(\operatorname{Int}(a)) \\
& \Longleftrightarrow(\forall b \in \mathbf{B}(X))(\operatorname{Int}(a) \subseteq b \rightarrow x \in b) \\
& \Longleftrightarrow(\forall b \in \mathbf{B}(X))((\forall y)(y \in \operatorname{Int}(a) \rightarrow y \in b) \rightarrow x \in a) \\
& \Longleftrightarrow(\forall b \in \mathbf{B}(X))((\forall y)((\exists c \in \mathbf{B}(X))(a \cup c=X \wedge y \notin c) \rightarrow y \in b) \rightarrow x \in b) \\
& \Longleftrightarrow(\forall b \in \mathbf{B}(X))((\forall y)(\forall c \in \mathbf{B}(X)(a \cup c=X \rightarrow y \in c \vee y \in b)) \rightarrow x \in b) \\
& \Longleftrightarrow(\forall b \in \mathbf{B}(X))((\forall c \in \mathbf{B}(X))(a \cup c=X \rightarrow(\forall y)(y \in c \vee y \in b) \rightarrow x \in b)) \\
& \Longleftrightarrow(\forall b \in \mathbf{B}(X))((\forall c \in \mathbf{B}(X))(a \cup c=X \rightarrow b \cup c=X) \rightarrow x \in b) \\
& \Longleftrightarrow(\forall b \in \mathbf{B}(X))(a \subseteq b \rightarrow x \in b) \\
& \Longleftrightarrow x \in C l(a)=a .
\end{aligned}
$$

(b) $\rightarrow(\mathbf{a})$. Let $\mathbf{B}(X) \subseteq R C(X)$. In order to show that $\mathbf{B}(X)$ is $U$-extensional let $a, b \in \mathbf{B}(X)$ with $a \nsubseteq \not b$ and $a \cup c=X$. We must show that $b \cup c \neq X$. The assumption (b) shows $C l(\operatorname{Int}(a)) \nsubseteq b$, which implies that there is an $x \in C l(\operatorname{Int}(a))$ with $x \notin b$. We obtain $\operatorname{Int}(a) \subseteq c$ implies $x \in c$ for all $c \in \mathbf{B}(X)$, and, hence, Int $(a) \nsubseteq b$. This implies the existence of a $y \in X$ such that $y \in \operatorname{Int}(a)$ and $y \notin b$. Again, we obtain that there is $c \in \mathbf{B}(X)$ such that $a \cup c=X$ and $y \notin c$, and, hence, $b \cup c \neq X$.

$(\mathbf{b}) \rightarrow(\mathbf{c})$. Let $\mathbf{B}(X) \subseteq R C(X)$. Then for any $a \cdot b \in \mathbf{B}(X)$ we have $a \cdot b=$ $C l(\operatorname{Int}(a \cdot b))$. Since $\cdot$ is a lattice meet we obtain that $a \cdot b \subseteq a, a \cdot b \subseteq b$, and, hence, $a \cdot b \subseteq a \cap b$. We conclude $a \cdot b=C l(\operatorname{Int}(a \cdot b)) \subseteq C l(\operatorname{Int}(a \cap b))$. For the converse inclusion, we have $C l(\operatorname{Int}(a \cap b)) \subseteq C l(\operatorname{Int}(a))=a$ and $C l(\operatorname{Int}(a \cap b)) \subseteq C l(\operatorname{Int}(b))=b$, and, hence, $C l(\operatorname{Int}(a \cap b)) \subseteq a \cdot b$.

$(\mathbf{c}) \rightarrow(\mathbf{b})$. Let $C l(\operatorname{Int}(a \cap b))=a \cdot b$. Then $a=a \cdot a=C l(\operatorname{Int}(a \cap a))=$ $C l(\operatorname{Int}(a))$, which shows that $\mathbf{B}(X) \subseteq R C(X)$.

$(\mathbf{b}) \rightarrow(\mathbf{d})$. Since (b) implies (c) we conclude that $(\mathbf{B}(X), \varnothing, X, \cup, \cdot)$ is in fact a sublattice of the Boolean algebra $R C(X)$. In order to show that $\mathbf{B}(X)$ is a dually dense in $R C(X)$, let $a \in R C(X)$ where $a \neq X$. Since $a=C l(\operatorname{Int}(a))$ 
and $\mathbf{B}(X)$ is a basis of the closed sets, there exists $c \in \mathbf{B}(X)$ such that $\operatorname{Int}(a) \subseteq c$. Furthermore, $a \neq X$ implies that there is an $x \notin C l(\operatorname{Int}(a))$, and, hence, $x \notin c$, which implies $c \neq X$. We conclude $a=C l(\operatorname{Int}(a)) \subseteq C l(c)=c$, which proves the assertion.

$(\mathbf{d}) \rightarrow(\mathbf{b})$. Obvious.

2. This follows immediately since all properties in 1 . are equivalent and imply (a) and (b).

We get the following corollary.

Corollary 2. Let $X$ be a topological space, $L=(L, 0,1,+, \cdot)$ be a lattice and let $h$ be an embedding of the upper semi-lattice $(L, 0,1,+)$ into the lattice $C(X)$ of closed sets of $X$. Suppose that the set $\mathbf{B}=\{h(a): a \in L\}$ forms a closed base for the topology of $X$. Then we have:

1. The following conditions are equivalent:

(a) $L$ is $U$-extensional.

(b) $\mathbf{B} \subseteq R C(X)$.

(c) For all $a, b \in L, h(a \cdot b)=C l(\operatorname{Int}(h(a) \cap h(b)))$.

(d) $h$ is a dually dense embedding of $L$ into the Boolean algebra $R C(X)$.

2. If any of the (equivalent) conditions (a),(b),(c) or (d) of 1. is fulfilled then:

(a) $L$ is a $U$-extensional distributive lattice.

(b) $X$ is a semi-regular space.

This corollary shows that if we require that a lattice $L$ be embeddable into the the Boolean algebra $R C(X)$ of some topological space $X$ with the properties of Corollary 2, then the lattice must be both distributive and U-extensional. In the next section we will show that this can be extended to $U$-extensional distributive contact lattices.

\section{Topological representation of distributive contact lattices}

The next theorem is the first main result of the paper.

Theorem 5. [Topological representation theorem for $U$-extensional distributive contact lattices]

Let $D=(D, 0,1,+, \cdot, C)$ be an $U$-extensional distributive contact lattice. Then there exists a semi-regular T0-space and a dually dense embedding $h$ of $D$ into the Boolean contact algebra $R C(X)$ of the regular closed sets of $X$.

Proof. Let $X=\operatorname{Clan}(D)$ be the set of all clans of $D$ and for $a \in D$, suppose $h(a)=\{\Gamma \in X: a \in \Gamma\}$. Using the properties of clans one can easily check that $h(0)=\varnothing, h(1)=X$ and that $h(a+b)=h(a) \cup h(b)$. This shows that the set $\mathbf{B}(X)=\{h(a): a \in D\}$ is closed under finite unions and can be taken as a closed basis for a topology of $X$. 
In order to show that $h$ is an embedding we must show that $a \leq b$ iff $h(a) \subseteq$ $h(b)$. From the left to the right this follows directly by the properties of clans. Suppose that $a \leq b$. Then there exists a prime filter $F$ such that $a \in F$ and $b \notin F$. Since prime filters are clans this shows that $h(a) \nsubseteq h(b)$. Consequently, $h$ is an embedding of the upper semi-lattice $(L, 0,1,+)$ into the lattice of closed sets $C(X)$ of the space $X$. By Corollary $2, X$ is a semi-regular space and $h$ is a dually dense embedding of $D$ into the Boolean algebra $R C(X)$.

Now, we want to show that $X$ is a T0-space. Let $\Gamma \neq \Delta$ be two different points (clans) of $X$; we will show that there exists a closed set $A$ containing exactly one of them. Suppose $\Gamma \nsubseteq \Delta$. Then there exists $a \in \Gamma$ with $a \notin \Delta$, and, hence, $\Gamma \in h(a)$ and $\Delta \notin h(a)$ so that the $A=h(a)$ will work. In the case $\Delta \nsubseteq \Gamma$ the assertion is shown analogously.

It remains to show that $h$ preserves the contact relation $C$, this is a direct consequence of Corollary 1.

Notice that Theorem 5 generalizes Theorem 5.1 from [4] to the distributive case. As a cosequence of Theorem 5 we obtain the following corollary, which has Theorem 2(2) as a special case. Recall that this theorem was already proved in $[3]$.

Corollary 3. [Topological representation theorem for $U$-extensional distributive lattices]

Let $D=(D, 0,1,+, \cdot)$ be an $U$-extensional distributive lattice. Then there exists a semi-regular T0-space and a dually dense embedding $h$ of $D$ into the Boolean contact algebra $R C(X)$ of the regular closed sets of $X$.

Proof. Since the overlap $O$ is a contact relation on $D$ the assertion follows immediately from Theorem 5 .

Due to Corollary 2 we already know that a representation in the sense of Theorem 5 for distributive contact lattices that are not $U$-extensional is not possible. As mentioned in the introduction we have to use weaker version of property that the image $h(D)$ of the embedding $h$ generates (or determines) the topology.

In order to prove such a representation theorem we consider "discrete" Boolean contact algebras defined in $[6]$ as follows. Let $(W, R)$ be a relational system where $W \neq \varnothing$, and $R$ is a reflexive and symmetric relation in $W$. Subsets of $W$ are considered as (discrete) regions and contact between two subsets $a, b \subseteq W$ is defined by $a \hat{C} b$ iff there is $x \in a$ and $y \in b$ such that $x R y$. Let $\mathbf{D}(W, R)$ denote the distributive lattice of all subsets of $W$ (which is, in fact, a Boolean algebra) with a contact $\hat{C}$ defined by $R$. It was shown in [6] that $\mathbf{D}(W, R)$ is indeed a Boolean and, hence, a distributive contact lattice. Since Boolean algebras are always $U$-extensional (and in addition, $O$-extensional) $\mathbf{D}(W, R)$ is a $U$-extensional distributive contact lattice. It is proved in [6] (using another terminology) that every Boolean contact algebra can be isomorphically embedded into an algebra of the above type. Inspecting the proof given in [6] one can see that it can be transferred easily to the distributive case. 
Theorem 6. Each distributive contact lattice $D=(D, 0,1,+, \cdot, C)$ can be isomorphically embedded into a Boolean contact algebra of the form $\mathbf{D}(W, R)$.

Proof. Let $W=\operatorname{Prime}(D)$ be the set of prime filters of $D$, let $F, G \in \operatorname{Prime}(D)$ and define $R$ as $F R G$ iff $F \times G \subseteq C$; Consider the Stone embedding $h_{\text {Prime }}$ : $D \rightarrow \operatorname{Prime}(D)$. It remains to show that $h$ preserves the contact relation. We observe that

$$
\begin{aligned}
& h_{\text {Prime }}(x) \hat{C} h_{\text {Prime }}(y) \\
& \Longleftrightarrow(\exists F, G \in \operatorname{Prime}(D))[x \in F, y \in G, \text { and } F \times G \subseteq C] \\
& \Longleftrightarrow x C y .
\end{aligned}
$$

Lemma 4

This completes the proof.

The following corollary is a direct consequence of the last theorem.

Corollary 4. [Extension lemma for distributive contact lattices]

Each distributive contact lattice can be embedded into a (U-extensional) Boolean contact algebra.

Now, we are ready to prove the second main result of this paper.

Theorem 7. [Topological representation theorem for distributive contact lattices]

Let $D=(D, 0,1,+, \cdot, C)$ be distributive contact lattice. Then there exists a semiregular T0-space, an embedding $h$ of $D$ into the Boolean contact algebra $R C(X)$ of the regular closed sets of $X$ and a embedding $k$ of $D$ into the Boolean algebra $R C(X)^{o p}$ so that $\{h(a): a \in D\} \cup\{k(a): a \in D\}$ is a sub-basis of the regular closed sets of $X$.

Proof. The proof can be realized in two steps. First, by Corollary 4, $D$ can be embedded into a ( $U$-extensional) Boolean contact algebra $B$. Let $e_{1}$ be the corresponding embedding. In the second step, we apply Theorem 5. Consequently, we get an embedding $e_{2}$ from $B$ into a semi-regular T0-space $X$. Now, let $h=e_{2} \circ e_{1}$, i.e. $h(a)=e_{2}\left(e_{1}(a)\right)$ and $k(a)=e_{2}\left(e_{1}(a)^{*}\right)\left(e_{1}(a)^{*}\right.$ is the complement (in $B$ ) of embedding of $a$ ). Then $h$ is as required. Since the set $\left\{e_{1}(a): a \in D\right\} \cup\left\{e_{1}(a)^{*}: a \in D\right\}$ generates the Boolean algebra $B$ we get the last assertion.

Next, we want to discuss the two representation theorems proved in this paper in more detail.

Discussion. 1. Notice that there is a difference in the usage of topologies in the topological representation Theorems 5 and 7 , and in the Stone topological representation theorems for distributive lattices and Boolean algebras. In Stone's theorem, topology is used to describe the image of the representation up to isomorphism. In our case, the topology is used to obtain good images of the elements of the lattice as regions, e.g., they should have a boundary, etc. For that reason Theorems 5 and 7 are just embedding theorems. In this respect they 
correspond much more to the embedding theorems for distributive lattices and Boolean algebras in algebras of sets. In our case, sets are replaced by regular closed sets.

2. If we consider contact structures as abstract "pointless" geometries, the question is which notion of points is suitable. In distributive contact lattices we may define two different kinds of points, i.e., prime filters and clans. Prime filters are in some sense "bad" points with respect to the contact structure. They correspond to the lattice part of the structure and can provide a representation by ordinary sets. It is possible to define a contact relation between those sets by means of the contact relation between points. Such a representation is constructed, for instance, in Theorem 6. Clans are "good points" with respect to the contact structure. They guarantee that the image $h(a)$ of each element of the lattice is a region, i.e., has a boundary, interior part, etc. The representation constructed in the proof of Theorem 7 can be interpreted as follows. In a first step we use "bad" points (prime filters) to represent the lattice as a lattice of sets ("bad" regions) and lift the contact relation to that structure. As a positive side-effect we end up with the property of $U$-extensionality. In the second step, the "good points" (clans) and $U$-extensionality are used to construct a representation with the intended topological properties. Since prime filters are clans they are among the "good points" of the second step, but they just appear in the interior part of the regions.

These informal explanations are reminiscent of considering prime filters and clans as atoms and molecules - the real points of the real spatial bodies. Similar ideas have been used in [5] for obtaining topological representation theorems for discrete versions of region-based theories of space.

\section{Conclusion and outlook}

In this paper we have generalized the notion of Boolean contact algebras by weakening the algebraic part to distributive lattices. This provided a deeper insight into the interaction of several notions used in the representation theory. As a result we obtained a characterization theorem for semi-regularity in topological spaces, which appeared as one of the main tools in the representation theory. We have given two representation theorems of such lattices in algebras of regular closed sets of some topological spaces, considered as standard models for region-based theory of space. These theorems are direct generalizations of some results from [6] and [4]. Because of the full duality of Boolean algebras, representations in algebras of regular closed sets and regular open sets are dual in that case. In fact, one can construct one representation in terms of the other by duality. In the distributive case duality is preserved only for the lattice part. Consequently, representations in algebras of regular open sets will need different techniques, which we plan to investigate in the future. Kripke semantics and associated reasoning mechanisms may also be developed (as in [1,11]). Another direction of research is to extend the vocabulary of distributive contact lattices with other mereotopological relations such as the non-tangential part-of, «, and 
dual contact, $\check{C}$. Last but not least, an open problem is the representation theory of a further generalization to non-distributive contact structures. First results of this direction can be found in [9]. Some non-topological representation theorems for non-distributive lattices may be found in [11]. The main problem here is that it is not obvious what kind of structure we want to consider as a standard model of a non-distributive contact lattice. Obviously, this question has to be resolved before the corresponding representation theory can be developed.

\section{References}

1. Allwein, G. and MacCaull, W. (2001). A Kripke semantics for the logic of Gelfand quantales. Studia Logica, 61:1-56.

2. Balbes, R. and Dwinger, P. (1974). Distributive Lattices. University of Missouri Press, Columbia.

3. Cornish, W. H. (1974). Crawley's completion of a conditionally upper continuous lattice. Pac J Math, 51(2):397-405.

4. Dimov, G. and Vakarelov, D. (2006). Contact algebras and region-based theory of space: A proximity approach. Fundamenta Informaticae. To appear.

5. Dimov, G. and Vakarelov, D. (2006). Topological Representation of Precontact algebras. In: W. MacCaull, M. Winter and I. Duentsch (Eds.), Relational Methods in Computer Science, LNCS No 3929, To appear.

6. Düntsch, I. and Vakarelov, D. (2006). Region-based theory of discrete spaces: A proximity approach. Discrete Applied Mathematics. To appear.

7. Düntsch, I. and Winter, M. (2005). Lattices of contact relations. Preprint.

8. Düntsch, I. and Winter, M. (2005). A representation theorem for Boolean contact algebras. Theoretical Computer Science (B), 347:498-512.

9. Düntsch, I. and Winter, M. (2006). Weak contact structures. In: W. MacCaull, M. Winter and I. Duentsch (Eds.), Relational Methods in Computer Science, LNCS No 3929:73-82.

10. Engelking, R., General topology, PWN, 1977.

11. MacCaull, W. and Vakarelov, D. (2001). Lattice-based Paraconsistent Logic. In: W. MacCaull, M. Winter and I. Duentsch (Eds.), Relational Methods in Computer Science, LNCS No 3929:178-189.

12. Stone, M. (1937). Topological representations of distributive lattices and Brouwerian logics. Časopis Pěst. Mat., 67:1-25.

13. Vakarelov, D., Düntsch, I., and Bennett, B. (2001). A note on proximity spaces and connection based mereology. In Welty, C. and Smith, B., editors, Proceedings of the 2nd International Conference on Formal Ontology in Information Systems (FOIS'01), pages 139-150. ACM.

14. Vakarelov, D., Dimov, G.,Düntsch, I. \& Bennett, B. A proximity approach to some region-based theory of space. Journal of applied non-classical logics, vol. 12, No3-4 (2002), 527-559

15. Wallman, H. (1938). Lattices and topological spaces. Math. Ann., 39:112-136. 